\title{
Association Between Nausea and Vomiting During Pregnancy and Sleep Quality: Mediating Effect of Depressive Symptoms
}

This article was published in the following Dove Press journal: International Journal of General Medicine

\author{
Pengsheng $\mathrm{Li}^{1,2, *}$ \\ Haiyan Wang ${ }^{1,2, *}$ \\ Gengdong Chen ${ }^{1,2}$ \\ Jinping Feng' \\ Dazhi Fan (1D ${ }^{1,2}$ \\ Dongxin Lin ${ }^{1,2}$ \\ Jiaming Rao ${ }^{1,2}$ \\ Zixing Zhou ${ }^{1,2}$ \\ Zhengping Liu (iD ${ }^{1,2}$ \\ Xiaoling Guo' \\ 'Department of Obstetrics, Affiliated \\ Foshan Maternity \& Child Healthcare \\ Hospital, Southern Medical University, \\ Foshan, People's Republic of China; \\ ${ }^{2}$ Foshan Fetal Medicine Research \\ Institute, Affiliated Foshan Maternity \& \\ Child Healthcare Hospital, Southern \\ Medical University, Foshan, People's \\ Republic of China \\ *These authors contributed equally to \\ this work
}

\begin{abstract}
Purpose: Nausea and vomiting during pregnancy (NVP) of varying degrees of severity are commonly experienced by pregnant women. This paper explores the association between NVP and poor sleep quality.

Patients and Methods: A cross-sectional study was conducted in an obstetrics clinic. A total of 2494 pregnant women (representing a response rate of 92.7\%) completed a selfadministered questionnaire.

Results: Of the 2494 participants, the mean sleep duration was 7.76 hours, and $54.3 \%$ of them report poor sleep quality (ie, a Pittsburgh Sleep Quality Index global score $>5$ ). In this study, $49.1 \%, 49.3 \%$ and $1.6 \%$ women reported mild, moderate, and severe NVP, respectively. Compare with women with mild NVP, women with moderate or severe NVP were more likely to report poor sleep quality $\left(\chi^{2}=30.16, p<0.001\right)$. After adjusted for demographics and gestational age, moderate and severe NVP were associated with poor sleep quality (adjusted odds ratio $(\mathrm{AOR})=1.66,95 \%$ confidence interval $(\mathrm{CI})=1.40-1.96$, and $\mathrm{AOR}=2.95,95 \% \mathrm{CI}=1.44-6.02$, respectively). Moreover, depressive symptoms mediated the association between NVP and poor sleep quality $(\beta=0.060, p=0.033,95 \% \mathrm{CI}=$ $0.028-0.180)$.
\end{abstract}

Conclusion: Our study suggested that moderate and severe NVP increase the risk of poor sleep quality. Further studies are warranted that focus on the mechanisms of the association between NVP and poor sleep quality.

Keywords: nausea, vomiting, pregnancy, sleep quality, depressive symptoms

\section{Introduction}

Nausea and vomiting are most commonly experienced during early pregnancy. In different samples from prior studies, the prevalence of nausea and vomiting of pregnancy (NVP) has been found to range from $35 \%$ to $91 \%{ }^{1-3}$ However, NVP symptoms are often ignored by pregnant women and clinicians, or pregnant women may refuse treatment for NVP because of concerns about the adverse effects of any treatment on their pregnancy. However, if NVP deteriorates into its severest form, hyperemesis gravidarum, it can threaten both mother and fetus. ${ }^{4,5}$ In addition, NVP can lead to a range of other mental and physical health problems, such as fatigue, anxiety, and depression, and thereby reduce overall quality of life. ${ }^{6-8}$

Sleep quality typically decreases during pregnancy. One meta-analysis reported the prevalence of poor sleep quality to be $45.7 \%$ (95\% CI: 36.5\% 55.2\%) among pregnant women. ${ }^{9}$ Furthermore, it has been found that women who experience poor
Correspondence: Xiaoling Guo

Department of Obstetrics, Affiliated Foshan Maternity \& Child Healthcare Hospital,

Southern Medical University, Foshan,

People's Republic of China

Email fsguoxl@163.com
International Journal of General Medicine 202I:14 4I-49

41

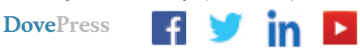

(c) (i) (5) $2021 \mathrm{Li}$ et al. This work is published and licensed by Dove Medical Press Limited. The full terms of this license are available at https://www.dovepress.com/terms.php C. you hereby accept the Terms. Non-commercial uses of the work are permitted without any further permission from Dove Medical Press Limited, provided the work is properly attributed. For permission for commercial use of this work, please see paragraphs 4.2 and 5 of our Terms (https://www.dovepress.com/terms.php). 
sleep quality are more likely to undergo preterm labor ${ }^{10}$ or a caesarean delivery. ${ }^{11}$ Previous research has also shown that poor sleep quality during pregnancy increases the risk of perinatal depression ${ }^{12}$ and suicide. ${ }^{13}$ NVP may be an important risk factor for reduction in sleep quality. A study conducted among lung cancer patients observed that serious vomiting was associated with poor sleep quality, ${ }^{14}$ while a case-control study conducted among pregnant women reported that hyperemesis gravidarum likewise increased the risk of poor sleep quality. ${ }^{15}$ However, most previous research has been carried out with reference to patients with hyperemesis gravidarum, and only a few studies to date have investigated the association between NVP and sleep quality in pregnant women who do not completely meet the diagnostic criteria of hyperemesis gravidarum.

Stressors and mental health status play important roles with regard to poor sleep quality during pregnancy. Specifically, somatic and psychological stressors during pregnancy can activate stress systems, such as a hypothalamic-pituitary-adrenal (HPA) axis stress response and inflammatory reaction. ${ }^{16}$ Prior research has concluded that NVP, as a somatic stressor, increases the risk of mental health problems such as depression and anxiety. ${ }^{8,17}$ Equally, depressive symptoms have been found to increase the risk of poor sleep quality during pregnancy. ${ }^{18}$ Accordingly, it is reasonable to infer that depressive symptoms may play an important role in the association between NVP and poor sleep quality.

We hypothesize that severe NVP increases the risk of poor sleep quality for pregnant women. To assess this postulation, we conducted a cross-sectional study to (a) examine the association between NVP and poor sleep quality and (b) evaluate the mediating effect of depressive symptoms on the association between NVP and poor sleep quality.

\section{Patients and Methods}

\section{Participants}

All participants were recruited from the Affiliated Maternal \& Child Health Hospital of Foshan, Southern Medical University. Pregnant women were eligible to participate if they were 18 years of age or older, were between weeks 6 and 28 of their pregnancy, and they planned to attend perinatal care and deliver at this hospital. With the help of trained interviewers, each woman completed a self-administered questionnaire. A total of 2494 women completed the questionnaire (representing a response rate of $92.7 \%$ ). All data were collected between November 2018 and March 2019. This study was approved by the Institutional Review Board of the Affiliated Maternal \& Child Health Hospital of Foshan, Southern Medical University, and was conducted in accordance with the guidelines of the 1975 Declaration of Helsinki. All participants provided written informed consent.

\section{Measures}

NVP

NVP was measured using the modified Pregnancy-Unique Quantification of Emesis and Nausea (PUQE) index. ${ }^{19}$ Compared to the original PUQE index, which focused on symptoms experienced during the preceding 12 hours (and was later modified to cover the previous 24 hours), ${ }^{20}$ the modified PUQE covers the first trimester of pregnancy. The modified PUQE index presents three subscales (questions referring to nausea, vomiting, and retching, respectively), and the score for each subscale ranges from 1 to 5 points. Thus, the total score from the modified PUQE index ranges from 3 to 15 points, with a higher score indicating more severe NVP. Lacasse et al provided the recommended cut-off total scores corresponding to "mild NVP" ( $\leq 6$ points), "moderate NVP" (7-12 points), and "severe NVP" ( $\geq 13$ points) in relation to the modified PUQE index. ${ }^{19}$ The modified PUQE index has been translated into Chinese and verified as demonstrating good reliability and validity. ${ }^{21}$

\section{Sleep Quality}

The Pittsburgh Sleep Quality Index (PSQI) was used to measure participants' sleep quality over the preceding 30 days. ${ }^{22}$ The duration of each night's sleep was also calculated. Across the PSQI's seven components, responses to each of which are rated from 1 ("no difficulty") to 3 ("severe difficulty"), the overall score can range from 0 to 21 points, with higher scores indicating poorer sleep quality. The PSQI has been translated to Chinese and verified to have good reliability and validity. In pregnant women, the cut-off score for identifying cases of poor sleep quality was $5 .{ }^{9}$

\section{Depressive Symptoms}

The Edinburgh Postnatal Depression Scale (EPDS) was used to measure depressive symptoms in participants over the preceding seven days. ${ }^{23}$ The EPDS was originally 
developed to screen for postpartum depressive symptoms, but the questions have been shown to be useful in measuring prenatal depressive symptoms too. ${ }^{24}$ Total scores from the 10-question EPDS can range from 0 to 30, with a higher score indicating more severe depressive symptoms. The EPDS has been translated to Chinese and verified to have good reliability and validity; in a Chinese adult population, the cut-off score for identifying depressive symptoms was $9 .^{25}$

\section{Covariates}

Demographical and gestational age data that have been reported as being associated with NVP or poor sleep quality were considered as covariates in the present study. Demographical data encompassing maternal age, education level, work status, household income per capita, and marital status were recorded via self-report. For example, education level was measured by asking each participant to specify the highest level of education that they had attained ("junior high school or below," "senior high school," or "college or university"). Work status was measured by asking participants if they were working, including full-time and part-time jobs, with their responses being either "working" or "not working." Household income per capita was calculated by dividing the total household income in the last year by the household's population. Marital status was measured by asking about the participant's current marital status, with their responses categorized as "married" or "unmarried or divorced."

\section{Statistical Analysis}

Sleep quality, NVP, depressive symptoms, and demographic data were assessed using frequency tables for categorical variables and compared using Pearson's chi-squared test. Maternal age and gestational age were summarized using means and standard deviations (SDs), and compared using a student's $t$-test. According to the recommended cut-off scores of the modified PUQE index, participants were divided into three groups (corresponding to mild NVP, moderate NVP, and severe NVP), while their PSQI scores were summarized with means and SDs and compared using analysis of variance. Logistic regression analysis was used to assess the association between NVP and sleep quality, and odds ratios (ORs) were obtained with $95 \%$ confidence intervals (CIs). The variables that were widely reported in previous studies or that were significant at the 0.10 level in the univariate logistic regression were entered into the multivariate logistic regression models. Three sets of logistic regression models were performed to evaluate the hypothesized associations. First, a univariate logistic regression model (Model 1) was run without being adjusted for the covariates. In Model 2, demographic data and gestational age were adjusted. Next, in Model 3, depressive symptoms and the covariates of Model 2 were adjusted. Structural equation modelling was used to measure the mediating effect of depressive symptoms on the association between NVP and poor sleep quality. The structural equation modelling was performed using the robust maximum-likelihood estimation. Standardized coefficients and indirect and total effects were estimated, and the $95 \%$ CI was measured using the bootstrap method with 2000 resamplings. All data were analyzed using SAS 9.2 (SAS Institute, Inc., Cary, NC) and Mplus 7.4 (Muthén and Muthén).

\section{Results}

\section{Participants}

Participants' demographic characteristics are detailed in Table 1. Of the 2494 participants who were analyzed, the mean age was 29.6 years old $(\mathrm{SD}=4.8$, range 18-46), and the mean gestational age was 22.9 (SD = 10.1, range 9-36) weeks. About half of the participants had graduated from college or university (52.0\%). Most of the participants were working $(75.0 \%)$ and were married $(95.4 \%)$.

\section{NVP}

The mean PUQE score of participants was 6.65 (SD = 2.56, range 3-15). According to the definitions of the modified PUQE, in this study, 49.1\%, 49.3\% and $1.6 \%$ women were evaluated to be experiencing mild, moderate and severe NVP, respectively.

\section{Sleep Quality}

The mean sleep duration of participants was $7.76(\mathrm{SD}=$ 1.38, range 3.00-13.00) hours, and 83.2\% participants sleep more 7 hours each day. The means global PSQI score was $6.39(\mathrm{SD}=3.18$, range $0-17)$, and $54.3 \%$ reported poor sleep quality (PSQI score $>5$ ). First trimester $(53.8 \%)$ and third trimester $(60.8 \%)$ pregnant women were more likely to report poor sleep quality than second trimester pregnant women $\left(48.3 \%, \chi^{2}=29.39, p<0.001\right)$. Compared with working women, non-working women were more likely to report poor sleep quality $\left(\chi^{2}=8.69\right.$, $p=0.003)$. 
Table I Characteristics of Participants and Sleep Quality ( $N=2494)$

\begin{tabular}{|c|c|c|c|c|c|}
\hline \multirow[t]{2}{*}{ Characteristics } & \multirow[t]{2}{*}{ Total n (\%) } & \multicolumn{2}{|c|}{ Poor Sleep Quality ${ }^{a}$} & \multirow[t]{2}{*}{$\chi^{2}$} & \multirow[t]{2}{*}{$p$} \\
\hline & & With n (\%) & Without n (\%) & & \\
\hline Total & $2494(100.0)$ & $1355(54.3)$ & $1139(45.7)$ & NA & NA \\
\hline Maternal age $(\text { years })^{\mathrm{b}}$ & $29.6(4.8)$ & $29.7(4.8)$ & $29.5(4.8)$ & 1.04 & 0.531 \\
\hline Gestational age (weeks) ${ }^{\mathrm{b}}$ & $22.9(10.1)$ & $23.5(10.3)$ & $22.1(9.8)$ & 1.49 & 0.137 \\
\hline Gestational age & & & & 29.39 & $<0.001$ \\
\hline First trimester & $617(24.8)$ & $332(53.8)$ & $285(46.2)$ & & \\
\hline Second trimester & $942(37.8)$ & $455(48.3)$ & 487 (5I.7) & & \\
\hline Third trimester & $935(37.4)$ & $568(60.8)$ & $367(39.2)$ & & \\
\hline Education level & & & & 4.12 & 0.127 \\
\hline Junior high school or below & $607(24.3)$ & $324(53.4)$ & $283(46.6)$ & & \\
\hline Senior high school & $584(23.4)$ & $300(51.4)$ & $284(48.6)$ & & \\
\hline College or university & $1297(52.0)$ & $729(56.2)$ & $568(43.8)$ & & \\
\hline Missing data & $6(0.2)$ & NA & NA & & \\
\hline Work status & & & & 8.69 & 0.003 \\
\hline Not working & $609(24.4)$ & $362(59.4)$ & $247(40.6)$ & & \\
\hline Working & $|87|(75.0)$ & $984(52.6)$ & $362(47.4)$ & & \\
\hline Missing data & $14(0.6)$ & NA & NA & & \\
\hline Household income per capita (CNY/month) & & & & 2.10 & 0.351 \\
\hline$\leq 3000$ & $452(18.1)$ & $234(51.8)$ & $218(48.2)$ & & \\
\hline $300 \mathrm{I}-7000$ & $963(38.6)$ & $538(55.9)$ & $425(44.1)$ & & \\
\hline$>7000$ & $1053(42.2)$ & $572(54.3)$ & $481(45.7)$ & & \\
\hline Missing data & $26(1.0)$ & NA & NA & & \\
\hline Marital status & & & & 1.59 & 0.208 \\
\hline Married & $2379(95.4)$ & $1285(54.0)$ & $1094(46.0)$ & & \\
\hline Unmarried or divorced & $108(4.3)$ & $65(60.2)$ & $43(39.8)$ & & \\
\hline Missing data & $7(0.3)$ & NA & NA & & \\
\hline Depressive symptoms ${ }^{c}$ & & & & 93.43 & $<0.001$ \\
\hline No & $1615(64.8)$ & $768(47.6)$ & $847(52.4)$ & & \\
\hline Yes & $854(34.2)$ & $580(67.9)$ & $274(32.1)$ & & \\
\hline Missing data & $25(1.0)$ & NA & NA & & \\
\hline
\end{tabular}

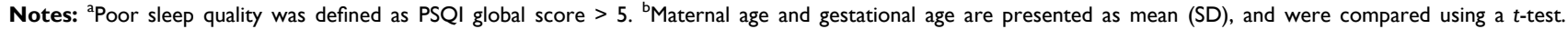
'Depressive symptoms was defined as EPDS score $\geq 9$.

Abbreviations: CNY, China Yuan; EPDS, Edinburgh Postnatal Depression Scale; NA, not applicable or no data available; PSQI, the Pittsburgh Sleep Quality Index.

\section{Depressive Symptoms}

The mean EPDS score of participants was 7.39 (SD = 4.14 , range $0-28$ ). There were $34.6 \%$ participants who reported depressive symptoms (EPDS score $\geq 9$ ). Women who were enduring poor sleep quality $(67.9 \%)$ were more likely to report depressive symptoms than women who had not experienced poor sleep quality $\left(32.1 \%, \chi^{2}=93.43, p<\right.$ $0.001)$. Women with moderate $(39.8 \%)$ and severe NVP $(40.0 \%)$ were more likely to report depressive symptoms than women with mild NVP $\left(29.2 \%, \chi^{2}=31.08, p<\right.$ 0.001).

\section{Association Between NVP and Sleep Quality}

Table 2 presents the relative sleep quality of participants alongside their varying degrees of NVP. As shown, there was no significant difference in sleep duration among women with different degrees of NVP $(F=0.61, p=$ 0.545). However, the global PSQI scores were higher in women with severe NVP than for women with mild or moderate NVP $(F=22.04, p<0.001)$. Of the seven PSQI components, women with severe NVP were higher in their scores for "subjective sleep quality", "sleep latency", 
Table 2 Self-Reported Sleep Quality by Nausea and Vomiting of Pregnancy ( $\mathrm{N}=2494)$

\begin{tabular}{|c|c|c|c|c|c|c|}
\hline \multirow[t]{2}{*}{ Characteristics } & \multirow[t]{2}{*}{ Total } & \multicolumn{3}{|l|}{$\mathbf{N V P}^{\mathbf{a}}$} & \multirow[t]{2}{*}{$\mathbf{F}$} & \multirow[t]{2}{*}{$p$} \\
\hline & & $\begin{array}{l}\text { Mild } \\
(n=\mid 225) \\
\text { Mean (SD) }\end{array}$ & $\begin{array}{l}\text { Moderate } \\
(n=\mid 229) \\
\text { Mean (SD) }\end{array}$ & $\begin{array}{l}\text { Severe } \\
(n=40) \\
\text { Mean (SD) }\end{array}$ & & \\
\hline Sleep duration (hour/day) & $7.76(1.38)$ & $7.73(1.34)$ & $7.79(I .4 I)$ & $7.74(1.6)$ & 0.61 & 0.545 \\
\hline Global PSQI score & $6.39(3.18)$ & $6(3.11)$ & $6.73(3.17)$ & $8.05(4)$ & 22.04 & $<0.001$ \\
\hline \multicolumn{7}{|l|}{ PSQI Components } \\
\hline Subjective sleep quality & I.3। (0.67) & $1.24(0.66)$ & $1.36(0.67)$ & $\mathrm{I} .48(0.78)$ & 11.50 & $<0.001$ \\
\hline Sleep latency & $1.15(1.01)$ & $1.08(1.01)$ & $1.20(1.01)$ & $1.58(1.17)$ & 8.28 & $<0.001$ \\
\hline Sleep duration & $0.59(0.87)$ & $0.58(0.85)$ & $0.59(0.88)$ & $0.70(1.11)$ & 0.36 & 0.700 \\
\hline Habitual sleep efficiency & $0.64(0.92)$ & $0.62(0.90)$ & $0.66(0.93)$ & $0.88(1.26)$ & 1.80 & 0.166 \\
\hline Sleep disturbance & I.II (0.60) & $1.07(0.59)$ & $1.13(0.61)$ & $\mathrm{I} .33(0.57)$ & 6.11 & 0.002 \\
\hline Use of sleeping medication & $0.01(0.10)$ & $0.01(0.11)$ & $0.01(0.10)$ & NA & NA & NA \\
\hline Daytime dysfunction & $1.60(0.91)$ & $\mathrm{I} .40(0.9 \mathrm{I})$ & $1.78(0.87)$ & $2.10(0.98)$ & 61.52 & $<0.001$ \\
\hline Poor sleep quality & $1355(54.3)$ & $600(49.0)$ & $726(59.0)$ & $29(72.5)$ & 30.16 & $<0.001$ \\
\hline
\end{tabular}

Notes: ${ }^{a}$ NVP was assessed using the Modified Pregnancy-Unique Quantification of Emesis and Nausea: mild NVP, < 6 points; moderate NVP, 7-I2 points; severe NVP, $\geq 13$ points. ${ }^{b}$ Poor sleep quality was defined as PSQI global score $>5$, and presented as numbers (\%). ${ }^{\mathrm{C}} \mathrm{A}$ chi-square test was used for poor sleep quality.

Abbreviations: NA, not applicable or no data available; PSQI, the Pittsburgh Sleep Quality Index; NVP, nausea and vomiting of pregnancy.

"habitual sleep efficiency", "sleep disturbance", and "daytime dysfunction" than were women with mild or moderate NVP $(p<0.05)$. There was no significant difference regarding the PSQI components of "sleep duration" and "use of sleep medication" among women with different degrees of NVP $(p>0.05)$. The prevalence of poor sleep quality among women who reported mild, moderate, and severe NVP was $49.0 \%, 59.0 \%$ and $72.5 \%$, respectively. Compared with women with mild NVP, women with moderate or severe NVP were more likely to report poor sleep quality $\left(\chi^{2}=30.16, p<0.001\right)$.

Logistic regression models were conducted to assess the association between NVP and sleep quality (see Table 3). Without adjusting any covariate (Model 1), compared with women with mild NVP, women with moderate or severe NVP were at higher risk of poor sleep quality: crude OR $(\mathrm{COR})=1.50,95 \% \mathrm{CI}=1.28-1.76$ and $\mathrm{COR}=$ $2.74,95 \% \mathrm{CI}=1.36-5.53$, respectively. After adjusting for maternal age, gestational age, educational level, work status, household income per capita, and marital status (Model 2), moderate and severe NVP were still associated with poor sleep quality: adjusted OR $(\mathrm{AOR})=1.66,95 \%$ $\mathrm{CI}=1.40-1.96$, and $\mathrm{AOR}=2.95,95 \% \mathrm{CI}=1.44-6.02$, respectively. In Model 2, we also examined the moderating effect of gestational age on the association between NVP and sleep quality. However, as there was found to be no significant moderating effect of gestational age $(p=$ 0.125), we inferred that there was no difference in the association between NVP and poor sleep quality across the three trimesters. In Model 3, after further adjusting for

Table 3 Association Between Nausea and Vomiting of Pregnancy and Sleep Quality

\begin{tabular}{|c|c|c|c|}
\hline Variates & $\begin{array}{l}\text { Model } I^{a} \\
(n=2494) \\
\operatorname{COR}(95 \% \mathrm{Cl})\end{array}$ & $\begin{array}{l}\text { Model } 2^{b} \\
(n=2457) \\
\text { AOR }(95 \% \mathrm{Cl})\end{array}$ & $\begin{array}{l}\text { Model } 3^{c} \\
(n=2432) \\
\text { AOR }(95 \% \mathrm{Cl})\end{array}$ \\
\hline \multicolumn{4}{|l|}{ NVP $^{d}$} \\
\hline Mild & 1.00 & 1.00 & 1.00 \\
\hline Moderate & $1.50(1.28,1.76)$ & $1.66(1.40,1.96)$ & $1.53(1.29,1.81)$ \\
\hline Severe & $2.74(1.36,5.53)$ & $2.95(1.44,6.02)$ & $2.69(1.30,5.55)$ \\
\hline
\end{tabular}

Notes: a Model I: Univariate logistic regression, not adjusted for covariate. ${ }^{b}$ Model 2: Adjusted for maternal age, gestational age, education level, work status, household income per capita, and marital status. 'Model 3: Adjusted for the covariates in Model 2 plus depressive symptoms. 'NVP was assessed using the Modified Pregnancy-Unique Quantification of Emesis and Nausea: mild NVP, < 6 points; moderate NVP, 7-12 points; severe NVP, $\geq 13$ points.

Abbreviations: AOR, adjusted OR; COR, crude OR; Cl, confidence interval; NVP, nausea and vomiting of pregnancy. 
Table 4 Mediating Effect of Depressive Symptoms on the Association Between NVP and Poor Sleep Quality $(\mathrm{N}=2432)$

\begin{tabular}{|l|l|l|l|}
\hline Characteristics & Estimate $^{\mathbf{a}}$ & $\mathbf{P}$ & $\mathbf{9 5 \%} \mathbf{C l}$ \\
\hline Path & & & \\
NVP $\rightarrow$ depressive symptoms & 0.053 & $<0.001$ & $0.033-0.087$ \\
Depressive symptoms $\rightarrow$ sleep quality & 1.127 & 0.007 & $0.682-2.954$ \\
NVP $\rightarrow$ sleep quality & 0.293 & $<0.001$ & $0.211-0.415$ \\
\hline Standardized effect & & & \\
Indirect effect & 0.060 & 0.033 & $0.028-0.180$ \\
Total effect & 0.353 & $<0.001$ & $0.263-0.480$ \\
\hline
\end{tabular}

Notes: ${ }^{a}$ Adjusted for maternal age, gestational age, education level, work status, household income per capita, and marital status. Model fit indices: $\mathrm{CFI}=0.992 ; \mathrm{RMSEA}=0.04 \mathrm{I}, 90 \% \mathrm{Cl}=0.038-0.043 ; \mathrm{SRMR}=0.027$.

Abbreviations: NVP, nausea and vomiting of pregnancy; Cl, confidence interval; CFI, comparative fit index; RMSEA, root mean square error of approximation; SRMR, standardized root mean square residual; Arrows, direction of effects.

depressive symptoms, NVP was still found to be associated with poor sleep quality, but the extent of this association was weaker.

\section{Mediating Effect of Depressive Symptoms}

The mediating effect of depressive symptoms on the association between NVP and poor sleep quality was further estimated in this study (see Table 4). The indirect effect of depressive symptoms was found to be significant $(\beta=$ $0.060, p=0.033,95 \% \mathrm{CI}=0.028-0.180)$, indicating that depressive symptoms partially mediated the association between NVP and poor sleep quality.

\section{Discussion}

Nausea and vomiting bring a substantial health burden to pregnant women, and can reduce their overall quality of life. Poor sleep quality is also a significant health problem during pregnancy that can increase the risk of pregnancy complication, including gestational diabetes mellitus ${ }^{26}$ and gestational hypertension. ${ }^{27}$ In this study, we found that severe NVP was associated with poor sleep quality among Chinese pregnant women.

In our study, $54.3 \%$ of the sample of pregnant women self-reported poor sleep quality. This result is consistent with the findings of previous research, 9,26 which collectively suggests that poor sleep quality is an issue for Chinese pregnant women. We found that there was no difference in sleep duration between pregnant women who reported mild, moderate, or severe NVP. However, NVP was found to be associated with poor sleep quality. Specifically, compared with women who reported mild NVP, those who reported moderate or severe NVP were more likely to suffer with poor sleep quality. This finding, too, is similar to those of previous studies. For example, Swallow et al reported that severe NVP increases the risk of insomnia among early pregnant women. ${ }^{28}$ Yildirım et al conducted a case-control study among 98 pregnant women, and reported that, compared with a health control group $(7.7 \%)$, women who were diagnosed with hyperemesis gravidarum were more likely to report poor sleep quality $(60.9 \%) .{ }^{15}$ In fact, our findings suggest that poor sleep quality is not limited to women with severe NVP who meet the diagnostic criteria of hyperemesis gravidarum, but is also found in women with moderate NVP. The biological mechanism of NVP is still not fully understood. Previous studies have suggested that the condition is associated with increased levels of reproductive hormones during pregnancy, such as estrogen and progesterone $^{29}$ and these hormones have also been found to be linked to sleep quality. ${ }^{30}$ Prior research has shown that high levels of estradiol increase the risk of restless legs syndrome and poor sleep quality among pregnant women. ${ }^{31}$ Also, in other animal studies, estrogen and progesterone have been observed to decrease the duration of non-rapid eye movement sleep and rapid eye movement sleep. ${ }^{32}$ Such evidence may partially explain the mechanism of the association between NVP and poor sleep quality, but more research is needed.

Previous studies have also shown that poor sleep quality is associated with gestational age and that poor sleep quality is more likely to be reported in the first and third trimesters. ${ }^{33,34}$ Our results in this regard are broadly consistent with those of prior research. However, in the present study, the moderating effect of gestational age on the association between NVP and sleep quality was not found to be significant. Such a finding suggested that there might 
be a long-term effect of NVP on sleep quality. Therefore, we further explored the role of mental health status on the association between NVP and poor sleep quality, and found that depressive symptoms mediated the association between them. Various earlier studies have suggested that NVP increases the risk of mental health problems in pregnancy, including depression, anxiety, ${ }^{35}$ and eating disorders. ${ }^{36}$ NVP is not only as an important stressor during pregnancy, but it also increases the risk of depression through psychological factors. Chou et al reported that NVP was positively correlated with perceived stress and maternal psychosocial adaptation, ${ }^{37}$ which have been found to increase the risk of perinatal depression. ${ }^{38,39}$ In addition, poor sleep quality is associated with mental health problems among pregnant women. A prospective longitudinal study reported that anxiety and depression is associated with poor sleep quality, and that this association is significant throughout the pregnancy. ${ }^{12}$ Most depressed patients experience disturbances of the HPA axis, such as hypercortisolemia and glucocorticoid receptor expression reduction. ${ }^{40}$ Numerous studies have suggested that disturbance of the HPA axis may be an important mechanism of sleep disorders in both pregnant $^{33}$ and non-pregnant individuals. ${ }^{41}$ The HPA axis's functions change during pregnancy, including a rise in cortisol concentrations rise and a blunting of diurnal rhythm. ${ }^{42}$ This in turn means that depressive symptoms during pregnancy may be more likely to cause sleep problems than in non-pregnant women. Therefore, depressive symptoms may at least partially explain the long-term effect of NVP on sleep quality.

Our findings suggest that NVP is associated with poor sleep quality, and this result has clinical implications because both NVP and sleep quality are modifiable factors. On the one hand, in perinatal care, more attention should be paid to screening for sleep problems and depression, especially in women with severe NVP. On the other hand, for women with severe NVP, effective interventions such as ginger, vitamin supplementation, fluid rehydration, and drug therapy should be taken to reduce NVP symptoms, ${ }^{29}$ and thereby potentially improve sleep quality during pregnancy.

The present study features several strengths, including, first, the fact that it was conducted among pregnant women, and not limited to hyperemesis gravidarum patients, which allowed us to explore the association between mild or moderate NVP and poor sleep quality. Second, sleep quality and NVP were measured using the
PSQI and the modified PUQE, respectively, which have been verified in terms of their reliability and validity among the Chinese population from which our study's sample was drawn. There are also several limitations to our study that should be noted as well. First, given the research's cross-sectional study design, it is difficult to make causal inferences. Second, as sleep quality was measured using a self-reporting questionnaire, rather than through objective records, recall bias cannot be completely ruled out. Third, due to the limitation of the study's outpatient clinic setting, data pertaining to potential confounding factors such as physical exercise and smoking were not collected and corrected in the multivariate analysis.

\section{Conclusion}

Our study's findings suggest that moderate and severe NVP increases the risk of poor sleep quality among Chinese pregnant women. Moreover, depressive symptoms were found to mediate the association between NVP and poor sleep quality. Therefore, more attention should be given to NVP, depressive symptoms, and sleep quality in prenatal care, and screening and intervention in respect of all three factors should be considered an important part of such care. Future research is needed in order to understand the mechanisms of the association between NVP and poor sleep quality. In particular, the psychological mediation processes associated with NVP, such as depression, anxiety, and eating disorders, should be considered in future research.

\section{Acknowledgments}

The authors express their gratitude to all the participants in this study.

\section{Funding}

This work was supported by Medical Science and Technology Foundation of Guangdong Province (No: C2019090); and Guangdong Basic and Applied Basic Research Foundation (No: 2019A1515111011).

\section{Disclosure}

The authors report no conflicts of interest in this work.

\section{References}

1. Chan OK, Sahota DS, Leung TY, Chan LW, Fung TY, Lau TK. Nausea and vomiting in health-related quality of life among Chinese pregnant women. Aust NZ J Obstet Gynaecol. 2010;50(6):512-518. doi:10.1111/j.1479-828X.2010.01216.x 
2. Einarson TR, Piwko C, Koren G. Quantifying the global rates of nausea and vomiting of pregnancy: a meta analysis. J Popul Ther Clin Pharmacol. 2013;20(2):e171.

3. Fejzo MS, Trovik J, Grooten IJ, et al. Nausea and vomiting of pregnancy and hyperemesis gravidarum. Nat Rev Dis Primers. 2019;5(1):62. doi:10.1038/s41572-019-0110-3

4. Fossum S, Vikanes AV, Naess O, Vos L, Grotmol T, Halvorsen S. Hyperemesis gravidarum and long-term mortality: a population-based cohort study. BJOG. 2017;124(7):1080-1087. doi:10.1111/14710528.14454

5. Mayer KH, McGill AL. Second-trimester fetal loss in a patient with hyperemesis gravidarum complicated by refeeding syndrome. Obstet Gynecol. 2019;133(6):1167-1170. doi:10.1097/AOG.000000 0000003276

6. Bai G, Korfage IJ, Groen EH, Jaddoe VWV, Mautner E, Raat H. Associations between nausea, vomiting, fatigue and health-related quality of life of women in early pregnancy: the Generation R study. PLoS One. 2016;11(11):e166133. doi:10.1371/journal.pone.0166133

7. Beyazit F, Sahin B. Effect of nausea and vomiting on anxiety and depression levels in early pregnancy. Eurasian J Med. 2018;50 (2):111-115. doi:10.5152/eurasianjmed.2018.170320

8. Bray N, Grasby KL, Lind PA, Painter JN, Colodro-Conde L, Medland SE. The psychosocial impact of nausea and vomiting during pregnancy as a predictor of postpartum depression. $J$ Health Psychol. 2019;1573745512. doi: $10.1177 / 1359105319859048$

9. Sedov ID, Cameron EE, Madigan S, Tomfohr-Madsen LM. Sleep quality during pregnancy: a meta-analysis. Sleep Med Rev. 2018;38:168-176. doi:10.1016/j.smrv.2017.06.005

10. Felder JN, Baer RJ, Rand L, Jelliffe-Pawlowski LL, Prather AA. Sleep disorder diagnosis during pregnancy and risk of preterm birth. Obstet Gynecol. 2017;130(3):573-581. doi:10.1097/AOG.0000000000002132

11. Teong A, Diong AX, Omar SZ, Tan PC. The impact of self-reported sleep on caesarean delivery in women undergoing induction of labour: a prospective study. Sci Rep. 2017;7(1):12339. doi:10.1038/ s41598-017-12410-7

12. Yu Y, Li M, Pu L, et al. Sleep was associated with depression and anxiety status during pregnancy: a prospective longitudinal study. Arch Womens Ment Health. 2017;20(5):695-701. doi:10.1007/ s00737-017-0754-5

13. Palagini L, Cipollone G, Masci I, et al. Stress-related sleep reactivity is associated with insomnia, psychopathology and suicidality in pregnant women: preliminary results. Sleep Med. 2019;56:145-150. doi:10.1016/j.sleep.2019.01.009

14. Akyuz RG, Ugur O, Elcigil A. Sleep quality in lung cancer patients. Asian Pac J Cancer Prev. 2013;14(5):2909-2913. doi:10.7314/ APJCP.2013.14.5.2909

15. Yildirim E, Demir E. The relationship of hyperemesis gravidarum with sleep disorders, anxiety and depression. J Obstet Gynaecol. 2019;39(6):793-798. doi:10.1080/01443615.2019.1572725

16. Palagini L, Gemignani A, Banti S, Manconi M, Mauri M, Riemann D. Chronic sleep loss during pregnancy as a determinant of stress: impact on pregnancy outcome. Sleep Med. 2014;15 (8):853-859. doi:10.1016/j.sleep.2014.02.013

17. Kjeldgaard HK, Eberhard-Gran M, Benth J', Vikanes ÅV. Hyperemesis gravidarum and the risk of emotional distress during and after pregnancy. Arch Womens Ment Health. 2017;20 (6):747-756. doi:10.1007/s00737-017-0770-5

18. Polo-Kantola P, Aukia L, Karlsson H, Karlsson L, Paavonen EJ. Sleep quality during pregnancy: associations with depressive and anxiety symptoms. Acta Obstet Gynecol Scand. 2017;96 (2):198-206. doi:10.1111/aogs.13056

19. Lacasse A, Rey E, Ferreira E, Morin C, Bérard A. Validity of a modified Pregnancy-Unique Quantification of Emesis and Nausea (PUQE) scoring index to assess severity of nausea and vomiting of pregnancy. Am J Obstet Gynecol. 2008;198(1):71. doi:10.1016/j. ajog.2007.05.051
20. Koren G, Magee L, Attard C, et al. A novel method for the evaluation of the severity of nausea and vomiting of pregnancy. Eur J Obstet Gynecol Reprod Biol. 2001;94(1):31-36. doi:10.1016/S03012115(00)00344-4

21. Yu-hua W, Lan W. Effect of health-related quality of life in women with nausea and vomiting of pregnancy (in Chinese). Clin J Med Officers. 2012;40(2):411-415.

22. Buysse DJ, Reynolds CFR, Monk TH, Berman SR, Kupfer DJ. The Pittsburgh Sleep Quality Index: a new instrument for psychiatric practice and research. Psychiatr Res. 1989;28(2):193-213. doi:10.1016/0165-1781(89)90047-4

23. Cox JL, Holden JM, Sagovsky R. Detection of postnatal depression. Development of the 10-item Edinburgh Postnatal Depression Scale. $\quad B r \quad J$ Psychiatr. 1987;150(6):782-786. doi:10.1192/ bjp. 150.6 .782

24. Howard LM, Ryan EG, Trevillion K, et al. Accuracy of the Whooley questions and the Edinburgh Postnatal Depression Scale in identifying depression and other mental disorders in early pregnancy. $B r \quad J$ Psychiatr. 2018;212(1):50-56. doi:10.1192/ bjp.2017.9

25. Zhao Y, Kane I, Wang J, Shen B, Luo J, Shi S. Combined use of the postpartum depression screening scale (PDSS) and Edinburgh postnatal depression scale (EPDS) to identify antenatal depression among Chinese pregnant women with obstetric complications. Psychiatr Res. 2015;226(1):113-119. doi:10.1016/j. psychres.2014.12.016

26. Wang H, Leng J, Li W, et al. Sleep duration and quality, and risk of gestational diabetes mellitus in pregnant Chinese women. Diabetic Med. 2017;34(1):44-50. doi:10.1111/dme.13155

27. Dunietz GL, Shedden K, Lisabeth LD, Treadwell MC, O'Brien LM. Maternal weight, snoring, and hypertension: potential pathways of associations. Am J Hypertens. 2018;31(10):1133-1138. doi:10.1093/ajh/ hpy085

28. Swallow BL, Lindow SW, Masson EA, Hay DM. Psychological health in early pregnancy: relationship with nausea and vomiting. $J$ Obstet Gynaecol. 2009;24(1):28-32. doi:10.1080/01443610310001620251

29. Bustos M, Venkataramanan R, Caritis S. Nausea and vomiting of pregnancy - What's new? Auton Neurosci. 2017;202:62-72. doi:10.1016/j.autneu.2016.05.002

30. Carrier J, Semba K, Deurveilher S, et al. Sex differences in age-related changes in the sleep-wake cycle. Front Neuroendocrin. 2017;47:66-85. doi:10.1016/j.yfrne.2017.07.004

31. Dzaja A, Wehrle R, Lancel M, Pollmacher T. Elevated estradiol plasma levels in women with restless legs during pregnancy. Sleep. 2009;32(2):169-174. doi:10.1093/sleep/32.2.169

32. Deurveilher S, Rusak B, Semba K. Female reproductive hormones alter sleep architecture in ovariectomized rats. Sleep. 2011;34 (4):519-530. doi:10.1093/sleep/34.4.519

33. Bublitz MH, Bourjeily G, D'Angelo C, Stroud LR. Maternal sleep quality and diurnal cortisol regulation over pregnancy. Behav Sleep Med. 2018;16(3):282-293. doi:10.1080/15402002.2016.1210147

34. Mindell JA, Cook RA, Nikolovski J. Sleep patterns and sleep disturbances across pregnancy. Sleep Med. 2015;16(4):483-488. doi:10.1016/j.sleep.2014.12.006

35. Mitchell-Jones N, Gallos I, Farren J, Tobias A, Bottomley C, Bourne T. Psychological morbidity associated with hyperemesis gravidarum: a systematic review and meta-analysis. BJOG. 2017;124(1):20-30. doi:10.1111/1471-0528.14180

36. Hoirisch-Clapauch S, Brenner B, Nardi AE. Adverse obstetric and neonatal outcomes in women with mental disorders. Thromb Res. 2015;135:S60-S63. doi:10.1016/S0049-3848(15)50446-5

37. Chou FH, Avant KC, Kuo SH, Fetzer SJ. Relationships between nausea and vomiting, perceived stress, social support, pregnancy planning, and psychosocial adaptation in a sample of mothers: a questionnaire survey. Int J Nurs Stud. 2008;45(8):1185-1191. doi:10.1016/j.ijnurstu.2007.08.004 
38. Lönnberg G, Jonas W, Unternaehrer E, Bränström R, Nissen E, Niemi M. Effects of a mindfulness based childbirth and parenting program on pregnant women's perceived stress and risk of perinatal depression-Results from a randomized controlled trial. J Affect Disord. 2020;262:133-142. doi:10.1016/j.jad.2019.10.048

39. Rodríguez MA, Valentine J, Ahmed SR, et al. Intimate partner violence and maternal depression during the perinatal period: a longitudinal investigation of Latinas. Violence Against Women. 2010;16(5):543-559. doi:10.1177/1077801210366959

40. Keller J, Gomez R, Williams G, et al. HPA axis in major depression: cortisol, clinical symptomatology and genetic variation predict cognition. Mol Psychiatry. 2017;22(4):527-536. doi:10.1038/ mp.2016.120
41. Antonijevic I, Antonijevic I. HPA axis and sleep: identifying subtypes of major depression. Stress. 2008;11(1):15-27. doi:10.1080/ 10253890701378967

42. Dickens MJ, Pawluski JL. The HPA axis during the perinatal period: implications for perinatal depression. Endocrinology. 2018;159 (11):3737-3746. doi:10.1210/en.2018-00677

\section{Publish your work in this journal}

The International Journal of General Medicine is an international, peer-reviewed open-access journal that focuses on general and internal medicine, pathogenesis, epidemiology, diagnosis, monitoring and treatment protocols. The journal is characterized by the rapid reporting of reviews, original research and clinical studies across all disease areas. The manuscript management system is completely online and includes a very quick and fair peer-review system, which is all easy to use. Visit http://www.dovepress.com/ testimonials.php to read real quotes from published authors.

Submit your manuscript here: https://www.dovepress.com/international-journal-of-general-medicine-journal 